\title{
Finite-aperture effects for Fourier transform systems with convergent illumination. Part II: 3-D system analysis
}

\author{
Damien P. Kelly ${ }^{\text {a }}$, Bryan M. Hennelly ${ }^{\text {a }}$, John T. Sheridan ${ }^{\text {a,* }}$, William T. Rhodes ${ }^{b}$ \\ ${ }^{a}$ UCD School of Electrical, Electronic and Mechanical Engineering, College of Engineering, Mathematical and Physical Sciences, University College \\ Dublin, Belfield, Dublin 4, Ireland \\ ${ }^{\mathrm{b}}$ Imaging Technology Center, Florida Atlantic University, 777 Glades Road, Bldg 43, Rm 486, Boca Raton, FL 33431, USA
}

Received 20 October 2005; received in revised form 23 January 2006; accepted 26 January 2006

\begin{abstract}
One of the most important optical signal processing operations is the optical Fourier transform (OFT). Of the arrangements for implementation of the OFT, perhaps the most flexible is that for the scaled optical Fourier transform (SOFT), as it allows control over the scale of the output Fourier transform distribution. By means of an analysis in cylindrical coordinates, we examine some of the practical limits introduced by the use of a thin lens of finite aperture in the implementation of the SOFT. Using simple rules of thumb that are based on an examination of the phase and magnitude deviations from the ideal (infinite-lens) diameter case, we define a volume inside the geometric shadow, which we refer to as a sub-geometric shadow. We then show that inside this sub-geometric shadow errors introduced by diffraction can be quantified.
\end{abstract}

(c) 2006 Elsevier B.V. All rights reserved.

OCIS: $070.0070 ; 070.2590 ; 050.1940$

\section{Introduction}

One of the fundamental operations in optical signal processing (OSP) is the optical Fourier transform (OFT). Various methods of implementing the OFT have been discussed [1-5]. Perhaps the most important, from a practical perspective, is the scaled OFT (SOFT), which allows the user to adjust the size of the output transform. An optical arrangement for the SOFT is illustrated in Fig. 1.

In his analysis, Goodman [1] assumes that the object is illuminated with a perfect converging spherical wave. However, the wave nature of light and the finite extent of the thin lens lead to diffraction effects that cause deviations from perfect spherical illumination. These diffraction effects can degrade the output OFT, and it would thus be beneficial if a sub-volume, or sub-geometric shadow, could

\footnotetext{
* Corresponding author. Tel.: +35301716 1927; fax: +353012830921. E-mail address: John.Sheridan@ucd.ie (J.T. Sheridan).

$U R L$ : http://www.ucd.ie/eleceng (J.T. Sheridan).
}

be identified where these effects remain below some quantifiable level. In Fig. 1 we illustrate what we mean by a subgeometric shadow identifying the apex point, $z_{c}$, on the principal axis.

In addressing this problem we begin by examining the deviations in the illumination field that occur inside the geometric shadow, comparing the case of a perfect spherical wave (ideal case) with the case of a spherical wavefront 'segment' propagating in the Fresnel-regime (non-ideal case). As in Part I of this paper [6], we present our analysis in a normalised coordinate system, thereby providing a more visually compact representation and making our results as general as possible. In our analysis of the deviations in the illumination field between the ideal (Goodman) and non-ideal (Fresnel) we show that [6-8]:

(i) The off-axis phase deviation extrema lie along welldefined analytically derived curves.

(ii) Large phase deviations occur on-axis. 


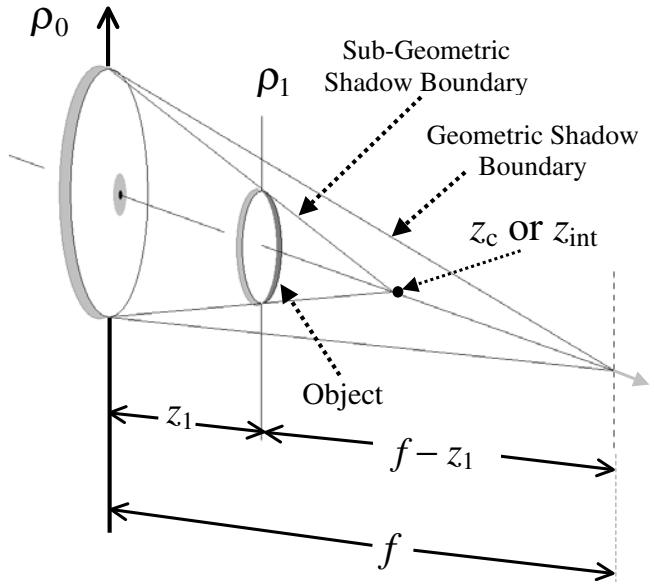

Fig. 1. 3-D cross-sectional representation of the scaled OFT system.

(iii) The magnitude deviation extremes appear to lie along the curves derived from the phase deviation analysis.

(iv) There are significant magnitude deviations on-axis. The nature of these deviations is different to the onaxis deviations that occur in Part I [6].

We note that, although the illuminating field phase and magnitude deviation analysis offers some insight into the effects of diffraction by the lens aperture, it is the intensity in the Fourier plane that in most cases is of primary interest. In order to examine the intensity deviations in the output Fourier plane we compare the intensity distributions predicted by the ideal and non-ideal cases in the Fourier plane for the case when our object (see Fig. 1) is a simple circular aperture.

The intensity deviations in the Fourier plane are examined using the 3-D version of the parameter we introduced in Part I [6], the Fourier Parameter, which allows us to estimate the deviation in intensity distribution over the entire plane. Once again this parameter can range in value from 0 to 2 , corresponding to an exact match and complete mismatch, respectively. Our approach to the 3-D analysis thus far follows Part I [6]. Out of necessity we depart in this paper from the previous approach in an attempt to clearly define a 3-D sub-geometric shadow. One of the reasons for this departure is the observation, that for a given SOFT system (with fixed lens diameter and focal length), illuminating fields of different wavelengths exhibit the same magnitude and phase distributions when examined at different (normalised) distances $(z)$ from the diffracting object. In the case of intensity deviations in the Fourier plane we show that:

(i) In keeping with the results presented in Part I [6] the intensity deviation extrema appear to lie on the curves that were derived from the phase deviation analysis.

(ii) Using the Fourier parameter error metric, and exploiting the relationship that exists between illuminating fields of different wavelengths, we can define a new sub-geometric shadow. If the SOFT is carried out inside this sub-geometric shadow the maximum error due to diffraction can be located and quantified.

From a practical point of view the most significant result in this work is presented in Section 3, where we specifically address the maximum error in the SOFT due to diffraction. Here we examine the analytic expression for intensity distribution in the Fourier plane for the ideal (Goodman) case when the object in question (see Fig. 1) is a simple circular aperture located at a normalised distance $z$ from the lens. The two main variables in this analytic expression are $K$ (which is defined explicitly in Section 2, and includes the focal length and radius of the lens, and the wavelength of the illuminating light) and $z$. It is found that a relationship exists such that for two given values of $K$ there exists two corresponding values for $z$, which produce identical, albeit scaled, intensity distributions. This relationship is examined numerically for the non-ideal (Fresnel) case and a similar relationship holds. We conclude: if the location and size of the maximum diffraction error for a particular SOFT system is found, this result can be generalised to any SOFT system.

\section{Deviations between ideal (Goodman) and non-ideal (Fresnel) illumination}

This section is broken up into four parts. In the first subsection we examine the phase deviations between the ideal and non-ideal case and identify a set of curves, which pass through the phase deviation extrema. In the second part we present some results from the phase analysis. In the third sub-section we examine the magnitude deviations and show that the magnitude deviation extrema also lie along the curves derived in the phase deviation analysis. Finally, in the last part we examine the on-axis magnitude deviations and compare them to the analogous deviations for the 2-D case [6].

\subsection{Phase analysis}

In this section the phase deviation extrema are located by employing standard calculus techniques outlined in Section 2 of Part I [6]. A thin spherical lens in the plane $z=0$, with aperture of radius $L$ and focal length $f$, is illuminated by a normally incident, unit-amplitude monochromatic plane wave of wavelength $\lambda$. Our concern is with the complex amplitude $U\left(z_{1}, \rho_{1}\right)$ of the resulting wave incident on (see Fig. 1) the object transparency in the plane $z=z_{1}$, where $\rho_{1}=\left(x_{1}^{2}+y_{1}^{2}\right)^{1 / 2}$ is the radial coordinate. Throughout our analysis we assume that Fresnel approximations are satisfied and neglect any constant phase factors.

If the aperture radius $L$ is sufficiently large that it can be ignored, the wave just to the right of the lens is given by

$U_{0}\left(0^{+}, \rho_{0}\right)=\exp \left(-\mathrm{j} \frac{k}{2 f} \rho_{0}^{2}\right)$, 
and the corresponding complex amplitude incident on the object by

$U_{S}\left(z_{1}, \rho_{1}\right)=\left(\frac{f}{f-z_{1}}\right) \exp \left[-\mathrm{j} \frac{k}{2\left(f-z_{1}\right)} \rho_{1}^{2}\right]$,

where $k=2 \pi / \lambda$. If the finite lens radius is taken into account, the complex amplitude incident on the object is given instead by the Fresnel diffraction integral [1-4]

$$
\begin{aligned}
& U_{F}\left(z_{1}, x_{1}, y_{1}\right)=\frac{1}{\mathrm{j} z_{1} \lambda} \int_{-\infty}^{\infty} \int_{-\infty}^{\infty} \operatorname{circ}\left(\frac{r}{L}\right) \\
& \quad \times \exp \left\{\frac{\mathrm{j} k}{2 z_{1}}\left[\left(x_{1}-x_{0}\right)^{2}+\left(y_{1}-y_{0}\right)^{2}\right]\right\} \mathrm{d} x_{0} \mathrm{~d} y_{0},
\end{aligned}
$$

where $\operatorname{circ}(r / L)=1$ for $r \leqslant L$ and 0 otherwise. Expressed in polar coordinates, Eq. (3) assumes the form

$$
\begin{aligned}
U_{\mathrm{F}}\left(z_{1}, \rho_{1}\right)= & \frac{2 \pi}{\mathrm{j} z_{1} \lambda} \int_{0}^{L} \exp \left\{\frac{\mathrm{j} k}{2}\left[\frac{\rho_{1}^{2}}{z_{1}}+\rho_{0}^{2}\left(\frac{1}{z_{1}}-\frac{1}{f}\right)\right]\right\} \\
& \times J_{0}\left(\frac{k \rho_{1} \rho_{0}}{z_{1}}\right) \rho_{0} \mathrm{~d} \rho_{0} .
\end{aligned}
$$

We wish to examine how the values given by Eqs. (2) and (4) differ from one another. Dividing the latter into the former gives

$$
\begin{aligned}
\frac{U_{\mathrm{F}}\left(z_{1}, \rho_{1}\right)}{U_{\mathrm{S}}\left(z_{1}, \rho_{1}\right)}= & F\left(z_{1}, \rho_{1}\right) \exp \left(\mathrm{j} \Delta \varphi^{\prime}\right) \\
= & \left(\frac{f-z_{1}}{f}\right) \frac{2 \pi}{\mathrm{j} z_{1} \lambda} \int_{0}^{L} \exp \left\{\frac { \mathrm { j } k } { 2 } \left[\frac{\rho_{1}^{2}}{z_{1}}+\rho_{0}^{2}\left(\frac{1}{z_{1}}-\frac{1}{f}\right)\right.\right. \\
& \left.\left.+\frac{\rho_{1}^{2}}{f-z_{1}}\right]\right\} J_{0}\left(\frac{k \rho_{1} \rho_{0}}{z_{1}}\right) \rho_{0} \mathrm{~d} \rho_{0},
\end{aligned}
$$

where $\Delta \varphi^{\prime}=\varphi_{U_{\mathrm{Fo} 0}\left(z_{1}, \rho_{1}\right)}-\varphi_{U_{\mathrm{s}}\left(z_{1}, \rho_{1}\right)}$. In order to be as general as possible the variables are normalised with respect to the physical parameters of the system giving

$$
\begin{aligned}
& \frac{2 K(1-z)}{\mathrm{j} z} \mathrm{e}^{\mathrm{j} K \frac{\rho^{2}}{z(1-z)}} \int_{0}^{1} \exp \left[\mathrm{j} K\left(\frac{P_{N}^{2}}{z}\right)(1-z)\right] \\
& \times J_{0}\left[K\left(\frac{2 P_{N} \rho}{z}\right)\right] P_{N} \mathrm{~d} P_{N},
\end{aligned}
$$

where $z=z_{1} / f, \rho=\rho_{1} / L, P_{N}=\rho_{0} / L$ and $K=\frac{k L^{2}}{2 f}$.

We note that $[4,9-11]$

$2 \int_{0}^{1} J_{0}(v P) \mathrm{e}^{\frac{-j u P^{2}}{2}} P \mathrm{~d} P=C(u, v)-\mathrm{j} S(u, v)$,

where $C(u, v)=2 \int_{0}^{1} J_{0}(v P) \cos \left(\frac{1}{2} u P^{2}\right) P \mathrm{~d} P$ and $S(u, v)=$ $2 \int_{0}^{1} J_{0}(v P) \sin \left(\frac{1}{2} u P^{2}\right) P \mathrm{~d} P$. It has been shown $[4,9,10]$ that this form of integral may be solved using Lommel functions, which are convergent [9-11] for all values of $u$ and $v$, and are defined as

$$
\begin{aligned}
& U_{n}(u, v)=\sum_{s=0}^{\infty}(-1)^{s}\left(\frac{u}{v}\right)^{2 s+n} J_{2 s+n}(v), \\
& V_{n}(u, v)=\sum_{s=0}^{\infty}(-1)^{s}\left(\frac{v}{u}\right)^{2 s+n} J_{2 s+n}(v) .
\end{aligned}
$$

Using integration by parts it can be shown that
$C(u, v)=\frac{\cos (u / 2)}{u / 2} U_{1}(u, v)+\frac{\sin (u / 2)}{u / 2} U_{2}(u, v)$,

$S(u, v)=\frac{\sin (u / 2)}{u / 2} U_{1}(u, v)-\frac{\cos (u / 2)}{u / 2} U_{2}(u, v)$.

It may also be shown that $C(u, v)$ and $S(u, v)$ can be written in a slightly different form

$$
\begin{aligned}
C(u, v)= & \frac{2}{u} \sin \left(\frac{v^{2}}{2 u}\right)+\frac{\sin (u / 2)}{u / 2} V_{0}(u, v) \\
& -\frac{\cos (u / 2)}{u / 2} V_{1}(u, v), \\
S(u, v)= & \frac{2}{u} \cos \left(\frac{v^{2}}{2 u}\right) \\
& -\frac{\cos (u / 2)}{u / 2} V_{0}(u, v)-\frac{\sin (u / 2)}{u / 2} V_{1}(u, v) .
\end{aligned}
$$

These forms are equivalent $[4,10,11]$. However, depending on whether $|u / v|>1$ or $|u / v|<1$, it is advantageous to use $V_{n}(u, v)$ or $U_{n}(u, v)$, respectively, because of the speed of numerical convergence of the solution [11]. The problem of diffraction from a circular aperture, under different illuminating conditions has also been discussed elsewhere [12].

We wish to find an analytical solution for Eq. (6). In order to do so we rearrange the equation so that it is in the same form as Eq. (7), where

$u=-2 K\left(\frac{1-z}{z}\right)$ and $v=2 K\left(\frac{\rho}{z}\right)$.

When analysing our system, we wish to examine the illuminated region only, i.e., the volume inside the geometric shadow. Noting that

$\left|\frac{u}{v}\right|=\left|\frac{1-z}{\rho}\right|$,

it is clear that if we wish to stay inside the illuminated area then $\rho<1-z$, thus Eq. (12) must be greater than unity. In this case the optimal solution to our equation, in terms of speed of convergence, is found using the Lommel $V$ series functions, i.e., Eq. (10).

Ideally, we would like to able to predict the locations where the extrema in phase deviation occur. As stated it is known $[4,10,11]$ that the $U$ and $V$ Lommel functions are equivalent. Therefore, although the $V$ series solutions converge more rapidly, it was decided to use the $U$ Lommel functions to analyse the phase deviation extrema due to the reduced complexity of the form of the solution. In this case the form of the solution to Eq. (6) is given by

$=\frac{K}{\mathrm{j} z}\left[I_{\mathrm{c}}(z, \rho)-\mathrm{j} I_{\mathrm{s}}(z, \rho)\right]$

with

$I_{\mathrm{s}}(z, \rho)=\sin \left[\frac{K \rho^{2}}{z(1-z)}\right] C(u, v)-\cos \left[\frac{K \rho^{2}}{z(1-z)}\right] S(u, v)$,
$I_{\mathrm{c}}(z, \rho)=\cos \left[\frac{K \rho^{2}}{z(1-z)}\right] C(u, v)+\sin \left[\frac{K \rho^{2}}{z(1-z)}\right] S(u, v)$. 
Adopting the approach taken in Section 2 of Part I [6], and simplifying the result in the manner outlined in Ref. [13], gives that stationary points lie along lines given by

$\rho=(2 N+1) \frac{\pi}{2} \frac{z}{4 K}$,

where $N \in N$. As mentioned we wish to examine the illuminated area only. Therefore, we rewrite Eq. (15) using normalised and scaled coordinates to give

$\rho^{\prime}=(2 N+1) \frac{\pi}{2}\left[\frac{z}{4 K(1-z)}\right]$,

where $\rho^{\prime}=\rho /(1-z)$.

\subsection{Phase deviation results}

Fig. 2(a) is a 3-D plot of the phase deviations between the ideal and non-ideal cases for $K=5 \pi / 8$ (see Eqs. (13) and (14)). The deviations in the plot range between $\pm \pi / 2$ and we note the presence of sharp on-axis phase oscillations. It is easier to appreciate the nature of these deviations by examining the contour plot in Fig. 2(b). We have chosen a low value of $K$ so that the rate of change of the deviations is slow. Physically a small $K$ value corresponds to a small lens with a focal length that is short compared to the wavelength. In order to identify trends we have performed many numerical searches on such phase deviation plots to: (i) determine the location of the phase deviation extrema (marked on this plot as white dots) and (ii) to determine the absolute phase deviation extremes for a range of positions in $z$ (marked on the plot as grey dots).

Following the analysis in Part I [6], a good approximation to the $z_{\mathrm{c}}$ point (cross-over point) is given by finding the point where the $N=2$ curve intersect the geometric shadow $\left(\rho^{\prime}=1\right)$. Rearranging Eq. (16) then gives

$z_{\mathrm{c}}=\frac{1}{1+\frac{5 \pi}{8 K}}$.
By following this simple rule of thumb Eq. (17) we identify the tip/apex of a sub-geometric shadow, which we refer to as SGS I, (Eq. (24) in Part I [6]). However, as is evident from Fig. 2, some large phase deviations occur on-axis inside SGS I. The fact that these deviations occur on the $z$-axis (principal axis) means that it is impossible to define a sub-geometric shadow that can exclude them.

\subsection{Magnitude analysis}

In Fig. 3(a), we present a 3-D plot of the difference in magnitude between the ideal and non-ideal cases. The largest magnitude variations occur on-axis and range between 0 and 2. The frequency of the oscillations decrease as $z$ approaches the Fourier plane. In Fig. 3(b) we present a contour plot of the magnitude deviations between the ideal and non-ideal case for $K=5 \pi / 8$. It can be seen that the even integer curves (Eq. (16)), derived from the phase deviation analysis, also pass through the local extrema.

\subsection{Magnitude analysis of on-axis points}

Allowing the variable $\rho$ in Eq. (13) to approach 0 and simplifying gives

$F(z, 0) \mathrm{e}^{\mathrm{j} \Delta \varphi^{\prime}}=1-\exp \left[\frac{-\mathrm{j} K(z-1)}{z}\right]$.

This result, Eq. (18), can be derived without recourse to a Lommel function-based solution. Setting $\rho=0$ in Eq. (6) causes it to reduce to the following integral

$$
\begin{aligned}
F(z, 0) \exp \left(\mathrm{j} \Delta \varphi^{\prime}\right)= & \frac{2 K(1-z)}{\mathrm{j} z} \\
& \times \int_{0}^{1} \exp \left[\mathrm{j} K\left(\frac{P_{N}^{2}}{z}\right)(1-z)\right] P_{N} \mathrm{~d} P_{N} .
\end{aligned}
$$

This integral is easily performed and the result is identical to Eq. (18). Fig. 4 is a plot of Eq. (18) for a value of $K=15.7$. As can be seen in the plot, and can be deduced
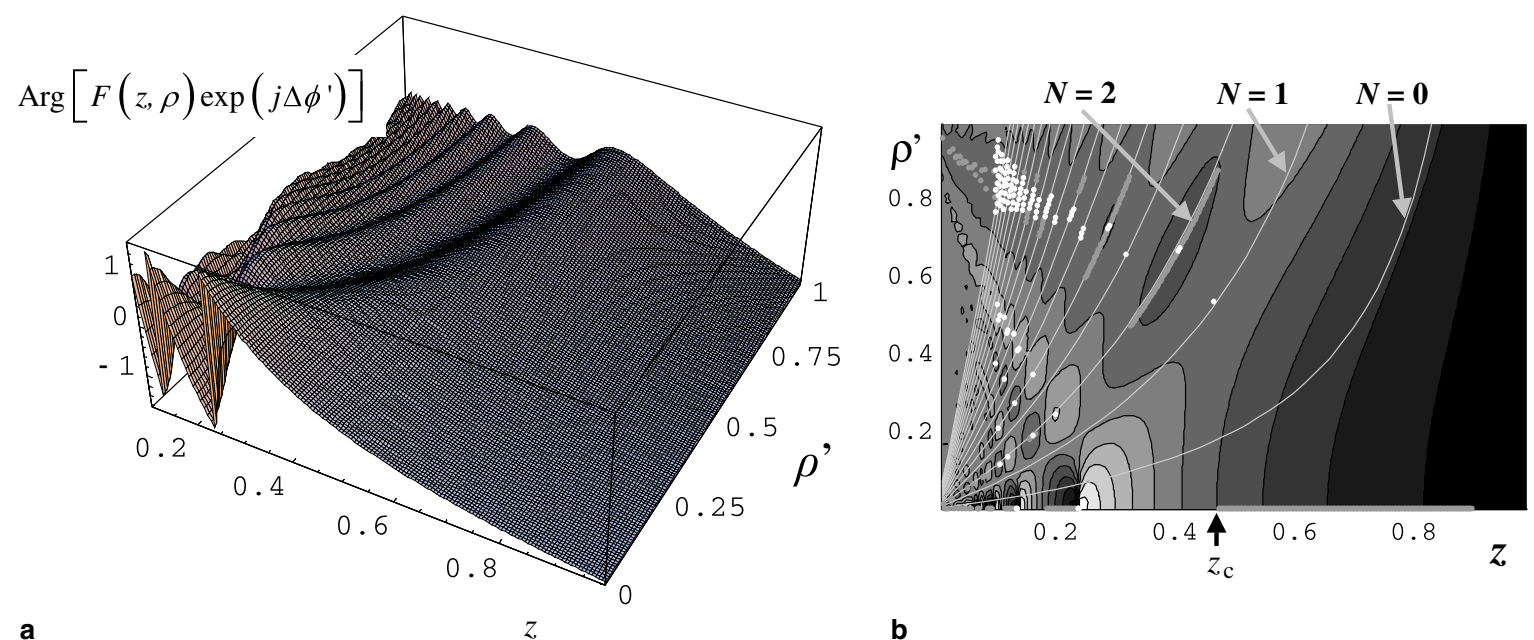

b

Fig. 2. (a) 3-D plot of phase deviations. (b) Phase deviations with curves for $N=0 \rightarrow 17$ (Eq. (16)), $K=5 \pi / 8$. 

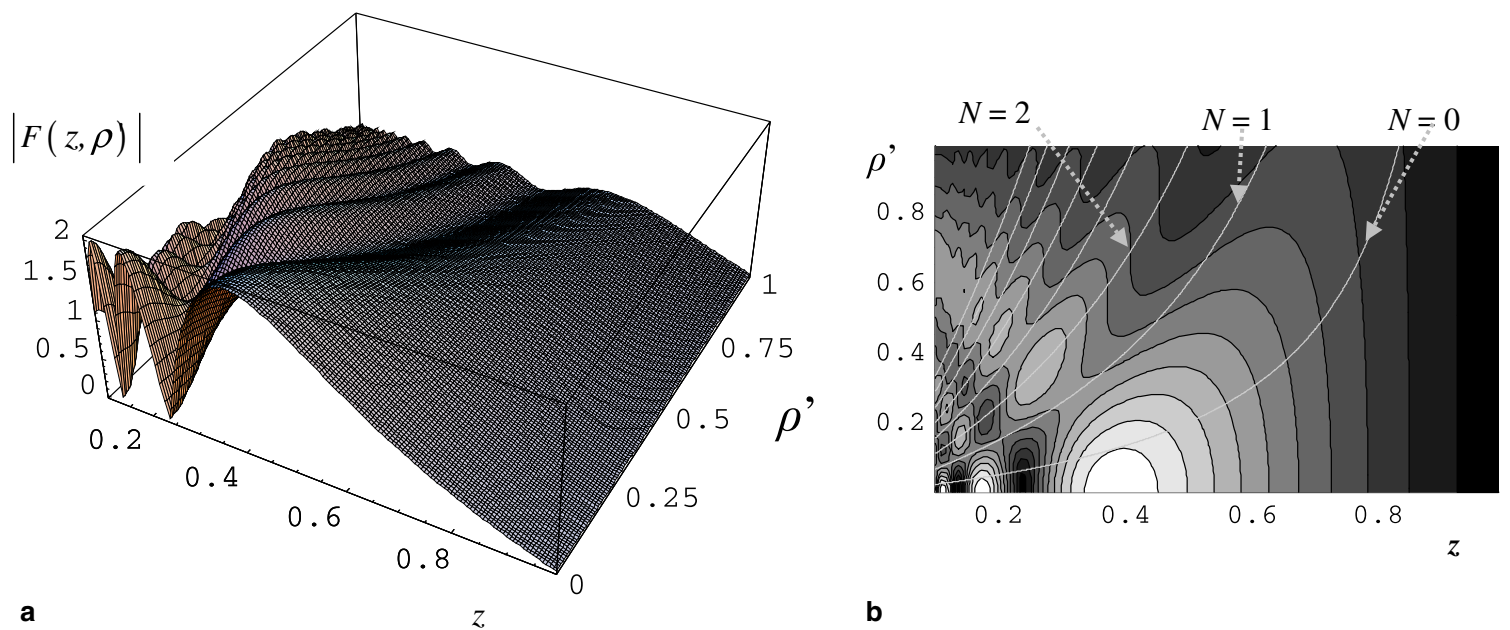

b

Fig. 3. (a) 3-D plot of magnitude deviations of $\left|F\left(z, \rho^{\prime}\right)\right|$. (b) Contour plot of magnitude deviations of $\left|F\left(z, \rho^{\prime}\right)\right|$ with curves for $N=0 \rightarrow 7$ (Eq. (16)), $K=5 \pi / 8$.

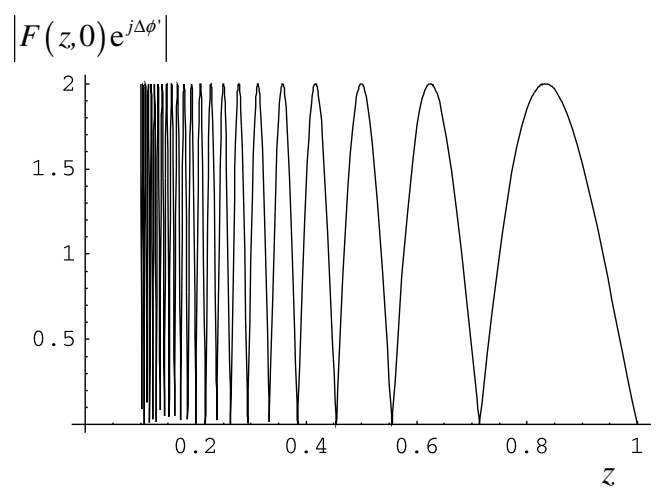

Fig. 4. Variation of $|F(z, 0)|$ (3-D cylindrical case) with $K=15.7$.

from examination of Eq. (18), the magnitude oscillates between 0 and 2. Furthermore the size of the magnitude deviations remains unaffected by $K$, although as $K$ increases the frequency of the oscillations increase.

We now return examining the on-axis magnitude deviations that occur in the 2-D case discussed in Part I of this paper. The on-axis solution for the 2-D case is given by integrating Eq. (17) in Part I [6] and setting $x^{\prime}=0$. This magnitude, referred to as $\left|F_{2 \mathrm{D}}(z, 0) \mathrm{e}^{\mathrm{j} \Delta \varphi^{\prime}}\right|$, is plotted in Fig. 5 for a $K$ value of 15.7. The difference between the plots in Figs. 4 and 5, is that in the latter, $\left|F_{2 \mathrm{D}}(z, 0) \mathrm{e}^{\mathrm{j} \Delta \varphi^{\prime}}\right|$ oscillates about a DC value of unity whereas the deviations in the 3-D case vary between 0 and 2. This has consequences when evaluating the maximum error limit for SGS-II [6] and is discussed further in Section 4. While it may seem surprising that the deviations on-axis are so different in nature for the circular and rectangular apertures, we note that similar on-axis deviations are predicted for diffraction from a simple circular aperture $[3,4,14]$ and experimentally demonstrated for a focused paraxial Gaussian beam [15].

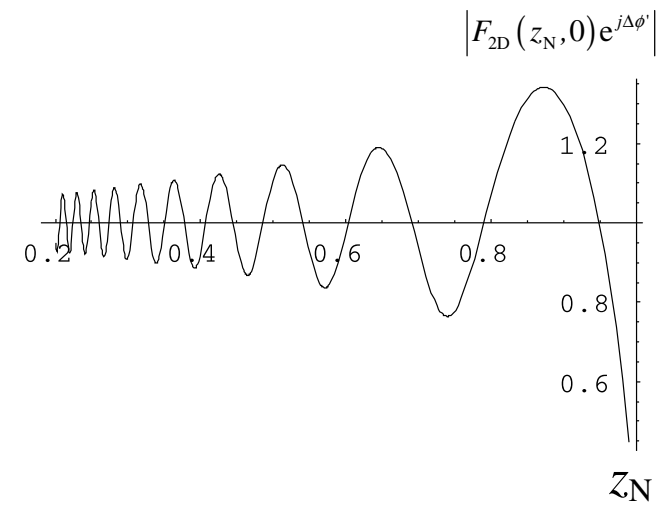

Fig. 5. Variation of $\left|F_{2 \mathrm{D}}\left(z_{\mathrm{N}}, 0\right) \mathrm{e}^{\mathrm{j} \Delta \varphi^{\prime}}\right|$ (2-D case) with $K=15.7$.

\section{Intensity analysis in the Fourier plane}

In the previous section the magnitude and phase deviations between an ideal converging spherical wave and a finite spherical wavefront propagated using the Fresnel transform were examined. Choosing a simple circular aperture as our object (see Fig. 1) we now examine the intensity deviations that occur in the Fourier plane between the ideal and non-ideal cases.

The metric we use, the Fourier parameter $[6-8,13]$, is a measure of the total difference in intensity at every point in the output plane (Fourier plane) between the ideal and non-ideal cases. This Fourier parameter has the advantage $[6-8,13]$ that a single percentage error value is calculated. It is examined for a range of object (circular aperture) positions, (assumed to be symmetric about the principal axis), and a range of aperture sizes.

We ensure that the power that enters the system for both the ideal and non-ideal cases is identical and throughout our analysis we have taken care to ensure that Parseval's theorem is obeyed (see Ref. [6, Section 4.1] for more 
details). We note that for the ideal case, when our object (circular aperture) is open to the edge of the geometric shadow, the power in the input (lens) plane and the Fourier plane is conserved. This is not true of the non-ideal case, as diffraction due to the lens aperture will cause the light to diffract outside of the geometric shadow and hence it will not pass through the fully illuminated object (circular aperture). Thus in the non-ideal case the initial power entering the system and the power in the Fourier plane cannot be the same, the "missing" power having been eliminated by the object (blocked by the aperture). Generally the larger the value of $K$ the smaller the diffractive effects are, and the greater the amount of power (non-ideal case) that is confined within the geometrical shadow. In Fig. 6 the percentage of power for a given $K$ that lies within the geometrical shadow is plotted as a function of $z$. It can be seen that for $K=15.7$ and at $z=0.9,75 \%$ of the initial power has been diffracted outside the geometrical shadow, whereas at the same distance, but with $K=157$ there is still over $95 \%$ of the initial power lying within the ideal illuminated cone.

We now proceed in Section 3.1 to examine and discuss the intensity deviations in the Fourier plane using the Fourier parameter. Then in Section 3.2 we identify the subgeometric shadow and outline how the maximum error due to diffraction is located and quantified.

\subsection{Intensity deviations in the Fourier plane for a simple circular window}

We now compare the output distributions in the Fourier plane predicted for the ideal and non-ideal systems when a circular aperture (object) of radius $a$, is placed inside the geometric shadow so that its centre point is on-axis and perpendicular to the $z_{1}$ axis. The output distributions are examined for a range of positions along the axis and for different aperture sizes. In order to be as general as possible we use the normalised coordinates: $z_{\mathrm{N}}=z_{1} / f$ and $a^{\prime}=a / L$, where $f$ is the focal length of the lens and $L$ is the radius of the lens. We use the variable $z_{\mathrm{N}}$ to emphasis that we are referring to the $z$ location of the object (circular aperture). In Section 2.1 we previously used scaled coordinates in

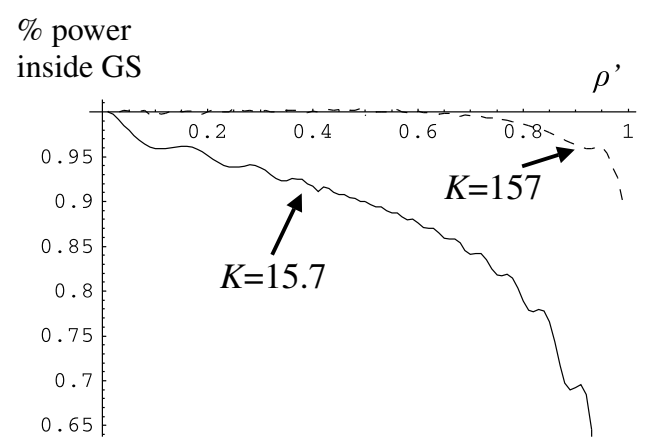

Fig. 6. Percentage of initial power inside geometric shadow as a function of $z$ for $K=15.7$ (solid line) and $K=157$ (dashed line). order to ensure that we always stayed within the illuminated region. Similarly, we now choose a scaled aperture size, $a_{\mathrm{N}}=a^{\prime} /\left(1-z_{\mathrm{N}}\right)$, which has a maximum value of unity when it is at the geometric shadow edge. For example if $a_{\mathrm{N}}=1$, the circular aperture (object) fully fills the geometric illuminated cone regardless of its position in $z_{\mathrm{N}}$.

The Fourier parameter was developed specifically to identify deviations in the shape of the output intensity distribution. We now extend it so that it is suitable for our 3-D analysis. For both models the intensity in the Fourier plane is normalised with respect to the power that has passed through the circular aperture (object). The resulting normalised intensities are then compared at every point in the Fourier plane and the accumulated error calculated. Therefore

$$
\begin{aligned}
& \Omega_{\mathrm{FP}}\left(a_{\mathrm{N}}, z_{\mathrm{N}}\right)= \\
& \quad \int_{0}^{2 \pi} \partial \theta \int_{-\infty}^{\infty}\left|\frac{I_{\mathrm{Good}}\left(a_{\mathrm{N}}, z_{\mathrm{N}}\right)}{\left.\operatorname{Power}_{\mathrm{Good}}\right|_{\text {Aperture }}}-\frac{I_{\text {Fresnel }}\left(a_{\mathrm{N}}, z_{\mathrm{N}}\right)}{\left.\operatorname{Power}_{\text {Fresnel }}\right|_{\text {Aperture }}}\right| \rho_{\mathrm{F}} \partial \rho_{\mathrm{F}},
\end{aligned}
$$

where the use of the $\partial \rho_{\mathrm{F}} \partial \theta$ notation underlines the fact that the cylindrical coordinate integral takes place in the Fourier plane.

Due to the complexity of Eq. (20) it is necessary to perform the integral numerically. In Fig. 7(a), a 3-D plot of Eq. (20) for a value of $K=50$ is presented. It can be seen that large deviations occur close to the axis and there is also a steady increase in the size of the deviations as $a_{\mathrm{N}}$ increases. Figs. 7(b) and 8 are contour plots of the Fourier parameter for values of $K=50$ and 157, respectively. A series of curves, given by Eq. (16) for values of $0 \leqslant N \leqslant 7$, are overlayed. As can be seen, the largest intensity deviations appear to lie close to the $N=2$ curve. Furthermore the deviations increase in size as one approaches the back Fourier plane. Comparing the two figures it can be seen that as $K$ increases, the intensity deviations appear to move closer together and move towards the Fourier plane.

Typically, if an aperture is large with respect to the wavelength of light, diffraction effects are less pronounced. Thus it is expected that as $K=L^{2} \pi /(\lambda f)$ increases, the errors due to diffraction should decrease.

In Fig. 9 we plot $\Omega_{\mathrm{FP}}\left(a_{\mathrm{N}}, z_{\mathrm{N}}\right)$ as $K$ increases, with $z_{\mathrm{N}}=0.5$ and $a_{\mathrm{N}}=1 . \Omega_{\mathrm{FP}}(0.5,0.7)$ decreases rapidly.

\subsection{Identification of the sub-geometric shadow}

One of the aims in this paper is to identify an optimal sub-geometric shadow such that errors due to diffraction can be identified and quantified. In Part-I [6] Section 4.2.2, a second sub-geometric shadow (SGS-II) was defined and the maximum error, due to diffraction for a given $K$ value, identified. Furthermore it was shown that the maximum error associated with SGS-II decreased as $K$ increased (see [6, Fig. 8, Part I]). To begin this approach was attempted for the 3-D case, however the maximum error associated with the new 3-D SGS-II did not decrease 

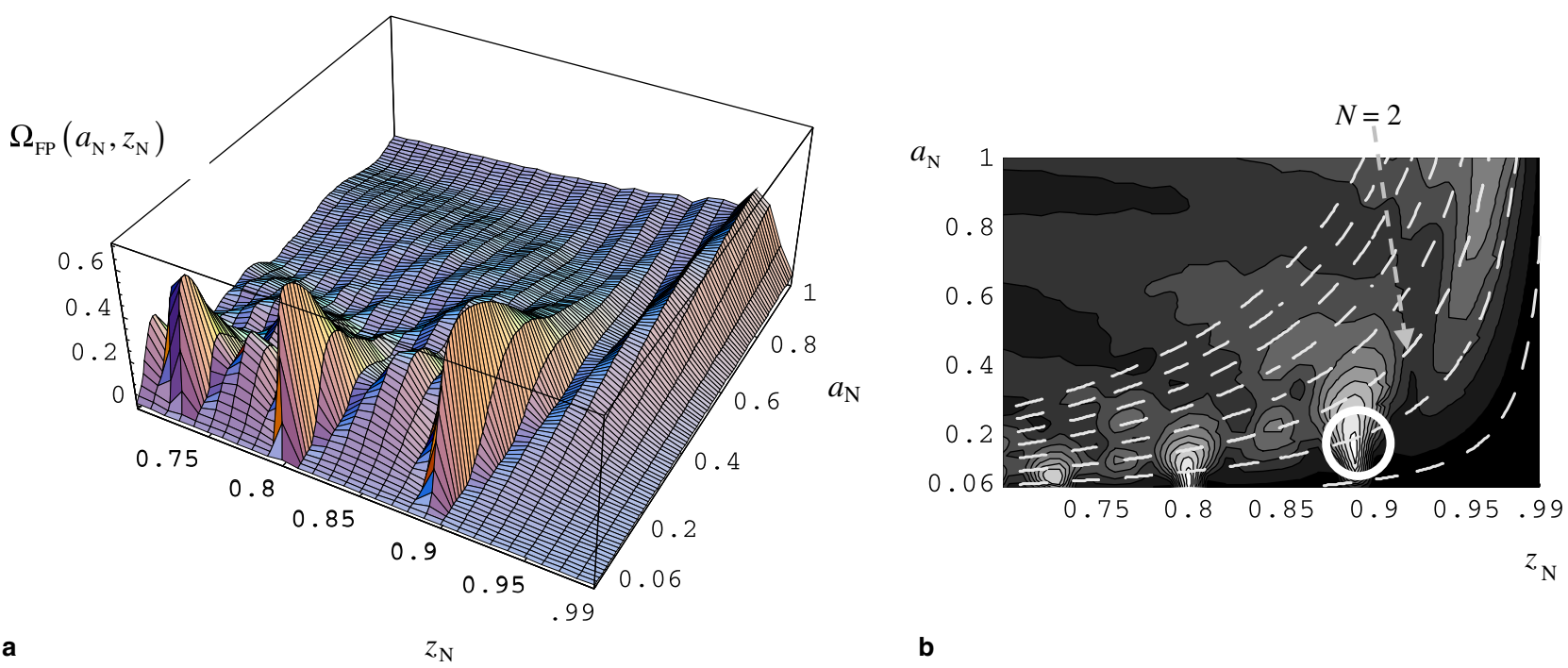

Fig. 7. (a) 3-D plot of variation of $\Omega_{\mathrm{FP}}\left(a_{\mathrm{N}}, z_{\mathrm{N}}\right)$ for $K=50$. (b) Contour plot of variation of $\Omega_{\mathrm{FP}}\left(a_{\mathrm{N}}, z_{\mathrm{N}}\right)$ for $K=50$.

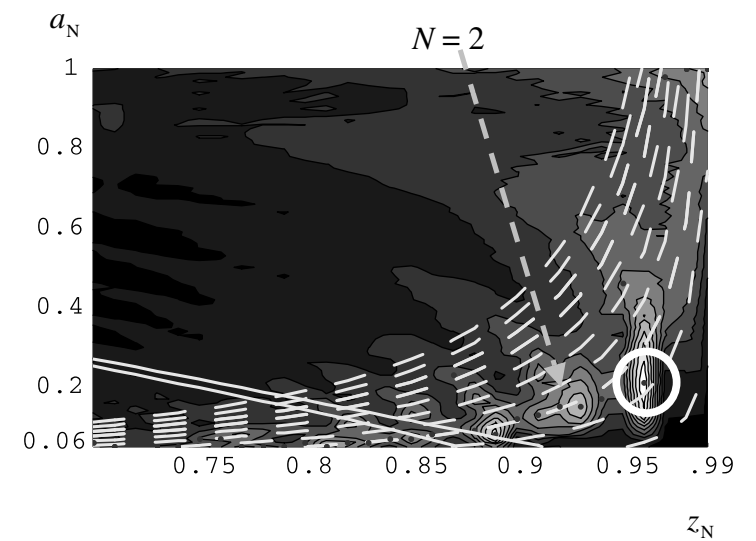

Fig. 8. Contour plot of variation of $\Omega_{\mathrm{FP}}\left(a_{\mathrm{N}}, z_{\mathrm{N}}\right)$ for $K=157$ (10 contour levels between 0 and 0.6 ).

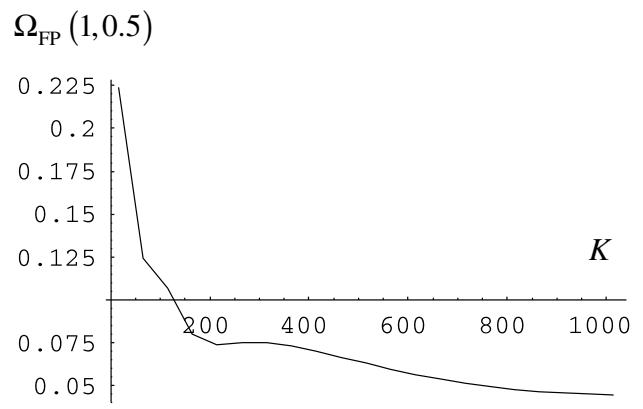

Fig. 9. Variation of $\Omega_{\mathrm{FP}}(1,0.5)$ with respect to $K$.

appreciable. There are several reasons for this the most significant being the large on-axis magnitude deviations. Furthermore numerical difficulties in calculating the Fourier Parameter for large $K$ values made validation difficult. For these reasons a different approach was developed.

We start by re-examining deviations between the ideal and non-ideal illumination fields; specifically Eqs. (6)-(14) and note that if these equations are written using the scaled parameter $\rho^{\prime}=\rho /(1-z)$, then the variable $K$ and $z$ always appear together in the form

$C(K, z)=K \frac{1-z}{z}$.

Note that once again we are referring to equations related to the deviations in the illuminating field, and therefore for clarity we use the parameter $z$ as opposed to $z_{\mathrm{N}}$. The scaling properties for diffracted and collimated waves are discussed in Ref. [16].

If Eq. (6), is rewritten in scaled coordinates, and Eq. (21) used, then

$$
\begin{aligned}
F\left(z, \rho^{\prime}\right) \exp \left(\mathrm{j} \Delta \varphi^{\prime}\right)= & \frac{2 C(K, z)}{\mathrm{j}} \mathrm{e}^{\mathrm{j} C(K, z) \rho^{\prime 2}} \int_{0}^{1} \exp \left[j\left(P_{\mathrm{N}}^{2}\right) C(K, z)\right] \\
& \times J_{0}\left[C(K, z)\left(2 P_{\mathrm{N}} \rho^{\prime}\right)\right] P_{\mathrm{N}} \mathrm{d} P_{\mathrm{N}} .
\end{aligned}
$$

Thus identical diffraction patterns will be observed for different $K$ values at different $z$ positions, so long as $C(K$, $z$ ) remains constant.

Let us take $K=15$ and $z=0.7$. Substituting these values into Eq. (21) gives that $C(K, z)=6.428$ (to three decimal places). The equivalent positions in $z$, so that identical diffraction patterns, can be observed is plotted in Fig. 10 as a function of $K$. It can be seen that as $K$ increases, $z$ also increases towards unity, its value at the Fourier plane.

While we have identified different locations in $z$ where the illuminating field incident on the object (circular aperture) for different values of $K$ is identical, we have not yet shown that this relationship extends to the deviations in the Fourier plane. We start by examining the ideal case. Fortunately an analytic solution exists, and since we are not concerned with phase in the Fourier plane we drop any phase factors to give 


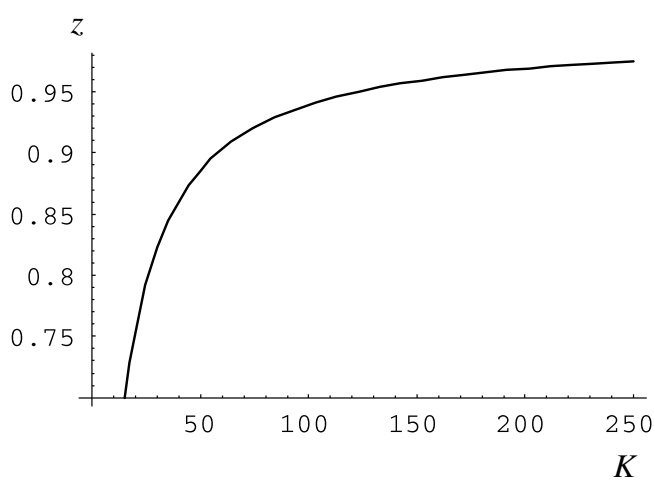

Fig. 10. Variation of Eq. (21) with $C(K, z)=6.428$.

$U_{\text {good }}\left(\rho_{\mathrm{F}}, K\right)=\frac{a_{\mathrm{N}}}{\mathrm{j} \rho_{\mathrm{N}}} J_{1}\left(a_{\mathrm{N}} \times \rho_{\mathrm{F}} \times K \times \chi\right)$,

where $\chi$ is a constant and $J_{1}(\cdot)$ is a Bessel function of first order. Varying $K$ results in a scaled version (with respect to the output variable $\rho_{\mathrm{F}}$ ) of the same distribution. Power must be conserved and so there is a corresponding change in the amplitude of the distribution such that Paresval's theorem holds.

Examining the non-ideal (Fresnel) case we refer to the analogous field in the Fourier plane as $U_{\text {fres }}\left(\rho_{\mathrm{F}}, K\right)$. In Fig. 11(a) and (b) we present a plot of the ideal (Goodman) and non-ideal (Fresnel) diffraction patterns for $K=157$ and 47 , respectively. In both cases the circular aperture (object) is fully open, so $a_{\mathrm{N}}=1$. For $K=157$ and 47 the object is placed at $z_{\mathrm{N}}=0.9606$ and 0.88 , respectively.

In order to test if scaling has occurred we divide $U_{\text {fres }}\left(\rho_{\mathrm{F}}\right.$, 47 ), the magnitude value at the "Comparison point" (see black circle in Fig. 11(a)), by $U_{\text {fres }}(0,47)$, giving a value of 7.84. The same calculation, carried out for $U_{\text {fres }}\left(\rho_{\mathrm{F}}, 157\right)$, gives a value of 7.80 indicating that the two distributions are almost exactly scaled versions of one another. This conclusion is given further weight by the fact that the corresponding Fourier parameters, calculated for these two cases, are 0.2275 and 0.2273 , respectively.

We still have not identified how we wish to quantify the maximum error for the 3-D case. Examining Fig. 7(a) and (b) $(K=50)$ we can see that the largest deviation occurs

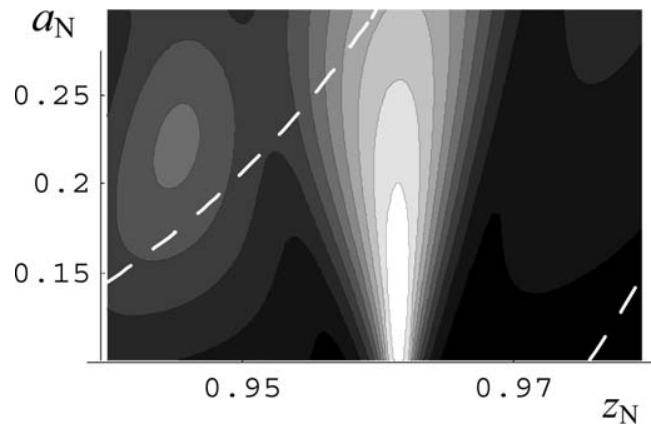

Fig. 12. Zoomed contour plot of Fig. 8. Variation of for $\Omega_{\mathrm{FP}}\left(a_{\mathrm{N}}, z_{\mathrm{N}}\right)$ $K=157$.

when the aperture is placed at $z_{\mathrm{N}}=0.88$ and $a_{\mathrm{N}}=0.16$. In this instance $C(K, z)=6.409$ (see Eq. (21)). If we wish to find the largest deviation when $K=157$, then the equivalent $z$ value can be found by solving Eq. (21). This yields a value of $z_{\mathrm{N}}=0.9606$. Since $a_{\mathrm{N}}$ is scaled with respect to $z_{\mathrm{N}}$ it has the same value. However, examining Fig. 8 the largest deviation appears to occur at $z_{\mathrm{N}}=0.96$ for $a_{\mathrm{N}}=0.2$. With further careful examination we find that this is only as a result of the limited resolution of the numerical calculation of the Fourier parameter for $K=157$. In Fig. 12, a higher resolution search over the range $0.94<z_{\mathrm{N}}<0.98$ and $0.1<a_{\mathrm{N}}<0.3$ reveals that in fact the largest deviation in this region is found at the location $z_{\mathrm{N}}=0.961$ and for $0.14<a_{\mathrm{N}}<0.16$.

In conclusion by numerically finding the largest deviations in the Fourier plane for a low $K$ value, it is possible to predict the magnitude and location of these deviations for larger and more realistic $K$ values. The clear advantage of performing the numerical search for a low value of $K$ is that the deviations are more easily observed.

\section{Conclusion}

We have examined the scaled optical Fourier transform (SOFT) for the 3-D case, assuming an apertured thin illuminating lens. Goodman [1] in his analysis assumes that

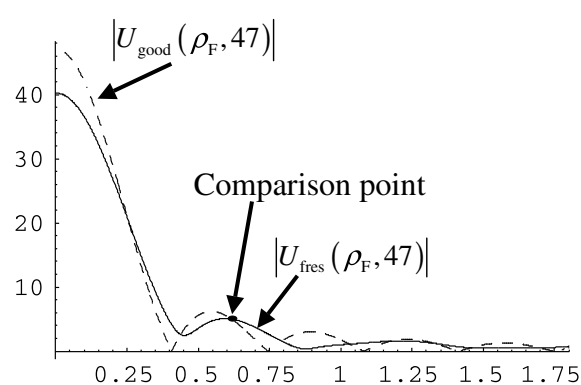

a

$100 \rho_{\mathrm{F}}$

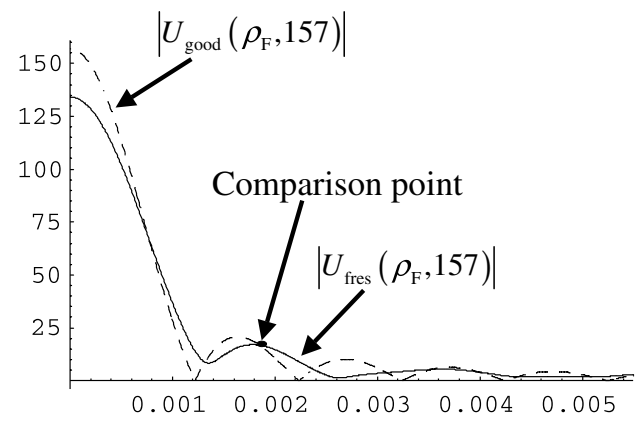

b
$\rho_{\mathrm{F}}$

Fig. 11. (a) Variation of $\left|U_{\mathrm{good}}\left(\rho_{\mathrm{F}}, 47\right)\right|$ (dashed) and $\left|U_{\mathrm{fres}}\left(\rho_{\mathrm{F}}, 47\right)\right|$ (solid) in the Fourier plane as a function of $100 \rho_{\mathrm{F}}$. (b) Variation of $\left|U_{\mathrm{good}}\left(\rho_{\mathrm{F}}, 157\right)\right|$ (dashed) and $\left|U_{\text {fres }}\left(\rho_{\mathrm{F}}, 157\right)\right|$ (solid) in the Fourier plane. 
the object is fully illuminated by a perfect converging spherical beam. We include the diffraction effects introduced by the lens aperture using the Fresnel transform with the aim of quantifying the resulting deterioration in the quality of the optical Fourier transform (OFT) performed. Based on our 2-D analysis, presented in Part I [6], we attempt to define a sub-volume or sub-geometric shadow, where the errors due to diffraction are quantifiable.

In Section 2 we examined the phase deviations between the ideal (Goodman) and non-ideal (Fresnel) illumination beams, and derived a set of analytic curves, which pass through the phase deviation extrema. While it was found that these curves pass close to the magnitude deviation extrema, it was also found that significant deviations (both magnitude and phase) occur on-axis for the 3-D case. In particular the nature of the 3-D on-axis magnitude deviations is quite unlike those observed in the 2-D simulations [6].

In Section 3, choosing a circular window as the object and applying the SOFT we examined the intensity deviations in the back Fourier plane as a function of object size and position. As in the 2-D case the intensity deviations appear along the set of curves derived for the phase case.

At this point we depart from the procedure described in Part I [6]. The intensity distribution in the Fourier plane, is a function of the wavelength of illuminating light, the focal length and diameter of the lens. Lumping these together using the parameter $K$, we can relate intensity deviations in the Fourier plane of different optical systems. Carrying out calculations for low values of $K$ ensures that the rate of change of deviations is slow and so the numerical operation can be performed more easily and accurately. Using Eq. (21) these results can then be extended to any given $K$ value.

In the analysis presented we have assumed aberration and noise free optics so that the fundamental error due to Fresnel diffraction can be identified. We note however that the Fresnel transform becomes increasingly inaccurate as the wavelength of illuminating light approaches the size of the diffracting aperture and also close to the object. To analyse more systems under these conditions it will be necessary to employ more accurate models for diffraction [1724].

\section{Acknowledgements}

We acknowledge the support of Enterprise Ireland and Science Foundation of Ireland through the Research Innovation and Proof of Concept Funds, the Basic Research and the Research Frontiers Programmes. We would also like to acknowledge the support of the Irish Research Council for Science, Engineering and Technology.

\section{References}

[1] J.W. Goodman, Introduction to Fourier Optics, third ed., Roberts and Co., Colorado, 2005.

[2] J.D. Gaskill, Linear Systems, Fourier Transforms, and Optics, Wiley, New York, 1978.

[3] E. Hecht, Optics, second ed., Addison-Wesley, Massachusetts, 1989.

[4] M. Born, E. Wolf, Principles of Optics, sixth ed., Pergamon Press Ltd., Oxford, 1980.

[5] R.N. Bracewell, The Fourier Transform and its Applications, McGraw-Hill, New York, 1965.

[6] D.P. Kelly, J.T. Sheridan, W.T. Rhodes, Opt. Commun. (in press), doi:10.1016/j.optcom.2006.01.047.

[7] D. Kelly, J.T. Sheridan, W.T. Rhodes, in: Frank Wyrowski (Ed.), Wave-Optical Systems Engineering II, Proc. SPIE, vol. 5182, 2003, p. 149 .

[8] D. Kelly, J.T. Sheridan, W.T. Rhodes, in: Frank Wyrowski (Ed.), Photon Management, Proc. SPIE, vol. 5456, 2004, p. 141

[9] A. Gray, G.B. Mathews, A Treatise on Bessel Functions and their Applications to Physics, MacMillian and Co. Ltd., London, 1922.

[10] G.N. Watson, A Treatise on the Theory of Bessel Functions, Cambridge University Press, London, 1922.

[11] K.D. Mielenz, J. Res. Natl. Inst. Stand. Technol. 103 (1998) 497.

[12] Q. Cao, J. Opt. Soc. Am. A 20 (2003) 661.

[13] D. Kelly, J.T. Sheridan, W.T. Rhodes, in: Bahram Javidi, Demetri Psaltis (Eds.), Optical Information Systems II, Proc. SPIE, vol. 5557, 2004, p. 279.

[14] J.E. Harvey, J.L. Forgham, Am J. Phys. 52 (1983) 243.

[15] G.P. Karman, A. van Duijl, M.W. Beijersbergen, J.P. Woerdman, Appl. Opt. 36 (1996) 8091.

[16] G.W. Forbes, Am. J. Phys. 62 (1994) 434.

[17] W.H. Southwell, J. Opt. Soc. Am. A 71 (1981) 7.

[18] G.W. Forbes, J. Opt. Soc. Am. A 13 (1996) 1816.

[19] M.W. Farn, J.W. Goodman, J. Opt. Soc. Am. A 7 (1990) 948.

[20] H. Osterberg, L.W. Smith, J. Opt. Soc. Am. A 51 (1961) 1050.

[21] C.J.R. Sheppard, P. Török, J. Opt. Soc. Am. A 20 (2003) 2156.

[22] C.J.R. Sheppard, Opt. Lett. 25 (2000) 1660.

[23] B. Richards, E. Wolf, Proc. Roy. Soc. (Lond.) A 253 (1959) 358.

[24] M.A. Alonso, A.A. Asatryan, G.W. Forbes, J. Opt. Soc. Am. A 16 (1999) 1958. 